\title{
High-dose intravenous immunoglobulin exerts neuroprotective effect in the rat model of neonatal asphyxia
}

\author{
Bo Chen', Jeong Seon Yoon ${ }^{2}$, Bingren $\mathrm{Hu}^{1}$ and Milan Basta ${ }^{3}$
}

BACKGROUND: Neonatal asphyxia is one of the leading causes of death in newborn and permanent neurological disabilities in surviving children. The underlying hypoxic-ischemic $(\mathrm{HI})$ injury triggers an inflammatory response lading to neuronal damage. Here, we tested the hypothesis that high-dose intravenous immunoglobulin (IVIG) could exert immunomodulatory effect in rat pups subjected to HI injury.

METHODS: $\mathrm{HI}$ injury was induced in 7-d-old pups by ligating the common carotid artery followed by exposure to $8 \%$ oxygen for $2 \mathrm{~h}$. Brain infarction was evaluated by imaging stained coronal brain sections. Neurological deficits were assessed in weeks 1 through 4 after HI. Western blotting and immunohistochemistry were used to assess complement fragment deposition in the brain tissue.

RESULTS: Treatment with IVIG at $2 \mathrm{~g} / \mathrm{kg}$ significantly and in a dose-responsive manner reduced brain infarction size as well as mortality and neurological deficits caused by HI. Anatomical and functional improvements in IVIG-treated pups correlated with decreased deposition of C3b complement fragments in the injured brain hemisphere.

CONCLUSION: IVIG significantly improved the outcome of HI injury in rat pups and could potentially be used for the treatment of human neonatal asphyxia to target proinflammatory complement fragments.

D espite major advances in monitoring and knowledge of newborn pathologies, neonatal asphyxia or hypoxic-ischemic encephalopathy (HIE), remains a serious condition that causes significant mortality and long-term morbidity. HIE is characterized by clinical and laboratory evidence of acute brain injury due to asphyxia. In the United States and in most technologically advanced countries, the incidence of this condition is one to eight cases per 1,000 births (1). HIE is the cause of $23 \%$ of all neonatal deaths worldwide; more than a million children who survive develop problems such as cerebral palsy, epilepsy, mental retardation, learning difficulties, and other disabilities (2). After a hypoxic-ischemic (HI) insult, a multifaceted cascade of events occurs that ultimately causes cell death and neurological damage. The various cascades include the complement system with subsequent augmentation of inflammation through induction of proinflammatory cytokines and chemokines; the production of reactive oxygen species leading to oxidative stress, and diminished mitochondrial function causing activation of apoptotic pathways. Complement activation has been implicated in the pathogenesis of HIE, both in rodent and human neonates. In 7-d-old rat pups, $\mathrm{HI}$ induced local complement cascade activation as early as $8 \mathrm{~h}$ after $\mathrm{HI}$. There was an eightfold increase in the activation fragment $\mathrm{C} 3 \mathrm{~b}$ at $16 \mathrm{~h}$. Both C3b and C9 were localized to injured neurons (3). In ELISA assays of cerebrospinal fluid obtained from 16 infants with HIE, the mean concentration of terminal complement complexes was elevated and the mean C9 concentration was diminished compared with cerebrospinal fluid from seven control infants (4). Here, we show that intravenous immunoglobulin (IVIG), when infused both before and after HI injury in 7-d-old rat pups, significantly reduced brain damage and subsequent neurological deficits as well as deposition of harmful complement fragments at the site of injury.

\section{RESULTS}

The Effect of IVIG and Vehicle on the Infarction Size

The average size of brain infarction induced by $\mathrm{HI}$ injury in our model was $32 \%$ of the ipsilateral hemispheric volume (Figure 1 and Figure 2a). Both pre- (30 min before ischemia) and posttreatment ( $1 \mathrm{~h}$ after hypoxia) with IVIG at $2 \mathrm{~g} / \mathrm{kg}$ significantly (by $43 \%$ and $47 \%$, respectively) reduced the size of brain infarction relative to HI only value (Figure 2a). Treatment with vehicle $(25 \mathrm{mmol} / \mathrm{l}$ glycine in the volume equivalent to the one in which IVIG was delivered) had no effect on the brain injury, since the infarction size in vehicle-treated animals did not differ significantly from animals subjected to HI only (Figure 2a). When groups of pups were treated with increasing doses of IVIG $(0.5,1$, and $2.0 \mathrm{~g} / \mathrm{kg})$ after hypoxia, a corresponding decrease in infarction size was observed, indicating that the protective effect of IVIG was exerted in a dose-responsive manner (Figure 2b).

\section{The Effect of IVIG on Mortality}

The overall mortality in this model of neonatal asphyxia was $25 \%$. All deaths occurred during the hypoxic phase. Therefore, any effect of IVIG treatment on fatality rate could only be assessed in pups pretreated with IVIG, since

'Shock, Trauma and Anesthesiology Research Center, Department of Anesthesiology, University of Maryland School of Medicine, Baltimore, Maryland; ${ }^{2}$ Laboratory of Neurosciences, National Institute on Aging Intramural Research Program, Baltimore, Maryland; ${ }^{3}$ BioVisions, Inc., Potomac, Maryland. Correspondence: Milan Basta (basta.milan@gmail.com) 
posttreatment was administered only to surviving animals ( $1 \mathrm{~h}$ after hypoxia). Pretreatment with IVIG, when compared with vehicle at the same volume, completely eliminated mortality (Table 1).
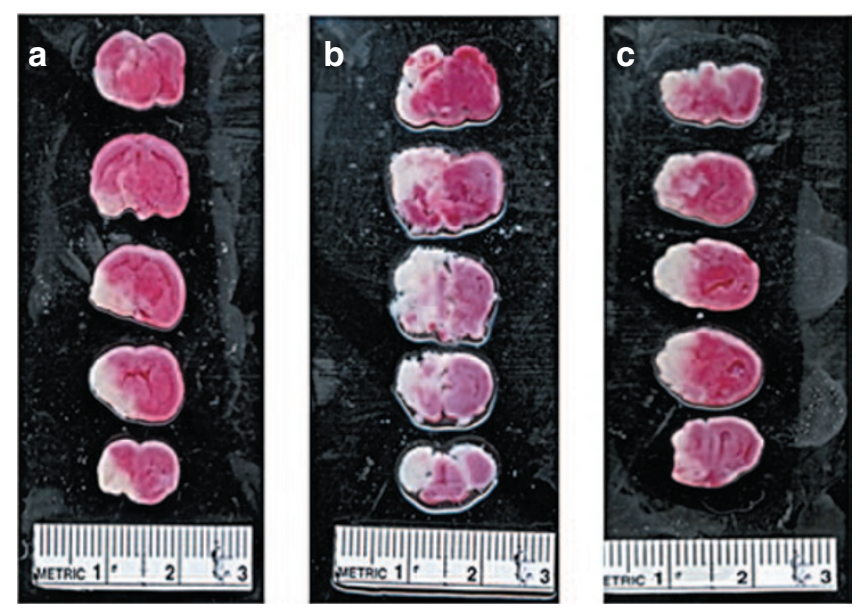

Figure 1. Brain infarctions following $\mathrm{HI}$ injury. (a-c) Brains from three pups subjected to hypoxic-ischemic (HI) injury, cut in $2 \mathrm{~mm}$ thick coronal sections and stained with 2,3,5-triphenyltetrazolium chloride monohydrate (TTC), showing varying degrees of infarction (white areas).
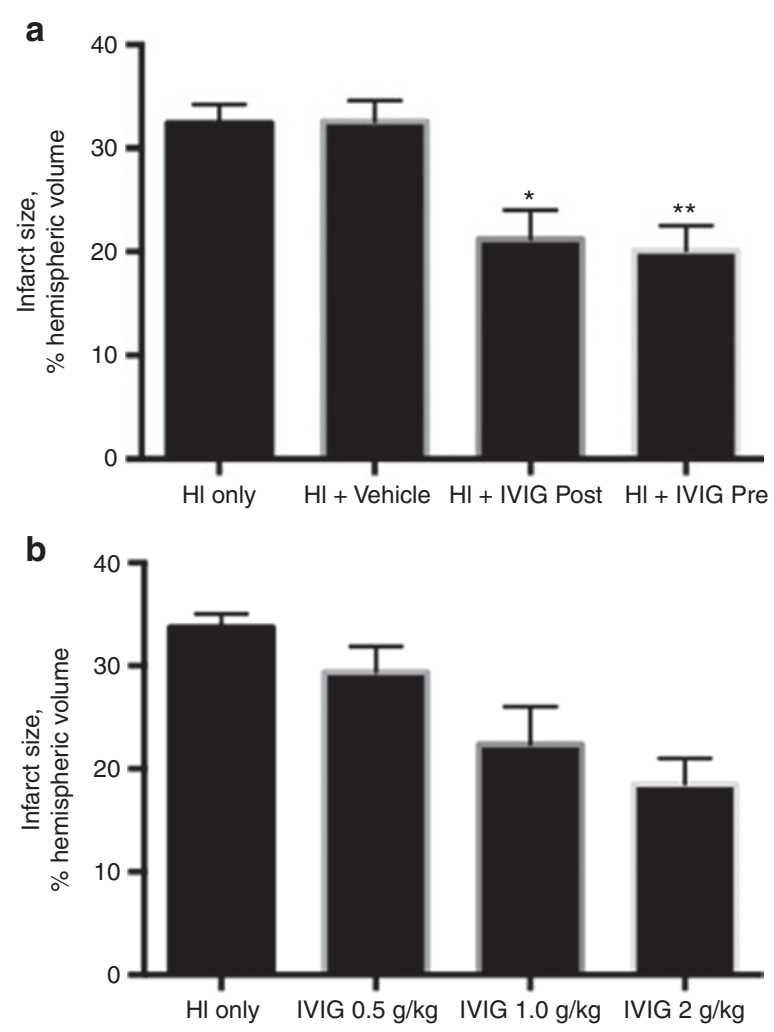

Figure 2. Intravenous immunoglobulin (IVIG) exerts dose-responsive neuroprotection before and after hypoxic-ischemic (HI) injury. (a) Seven-dayold pups were subjected to $\mathrm{HI}$ alone $(\mathrm{HI}$ alone, $n=25)$, treatment with IVIG vehicle after hypoxia (HI+Vehicle, $n=14)$, IVIG at $2 \mathrm{~g} / \mathrm{kg}$ before ischemia (HI+IVIG Post, $n=18$ ), and IVIG at $2 \mathrm{~g} / \mathrm{kg}$ after hypoxia (HI+IVIG Pre, $n=$ 15); ${ }^{*} P<0.01,{ }^{* *} P<0.001 \mathrm{IVIG}$ vs. vehicle. (b) Brain infarction size in pups treated with increasing doses of IVIG at $0.5 \mathrm{~g} / \mathrm{kg}(n=9), 1 \mathrm{~g} / \mathrm{kg}(n=10)$, and $2 \mathrm{~g} / \mathrm{kg},(n=12)$ posthypoxia.

\section{Neurological Deficit in Animals Treated With IVIG}

Neurological deficits were evaluated both early $(24 \mathrm{~h}, 48 \mathrm{~h}, 72 \mathrm{~h}$, and $7 \mathrm{~d}$ ) and late (2, 3, and $4 \mathrm{wk}$ ) after HI injury and HI+IVIG/ vehicle treatment. Motor dysfunction was significantly lower 1-7 $\mathrm{d}$ after $\mathrm{HI}$ in IVIG-treated pups, as evaluated by a $0-4$ neurological deficit scale as shown in Figure 3a. Likewise, IVIG treatment reduced neurological deficit in weeks 2-4 after HI (periods equivalent to those when permanent neurological damage is observed in children surviving neonatal asphyxia). The wire hang test, which evaluates neuromuscular and locomotor function, was used at 2, 3, and 4 wk after HI injury. At each time point of evaluation, IVIG-treated group showed significant motoric function improvement (around $55 \%)$ when compared with nontreated and vehicle-treated pups (Figure $3 \mathbf{b}$ ). Righting reflex test was applied at $2 \mathrm{wk}$ after HI injury. This test is believed to be a reflection of subcortical function. As shown in Figure 3c, IVIG treatment at $2 \mathrm{~g} / \mathrm{kg}$ significantly reduced the latency period of the righting reflex in comparison with HI only and vehicle-treated pups. Foot fault (grid walk) is a test of motor coordination. Pups were evaluated at $4 \mathrm{wk}$ after $\mathrm{HI}$ and, as shown in Figure 3d, IVIG treatment improved the motoric coordination by over $62 \%$ when compared with vehicle treatment.

\section{Complement Activation at the Site of Injury and Its Attenuation With IVIG}

The levels of the active fragment of the third complement component, C3b, were evaluated by western blot in brain samples of pups subjected to vehicle and IVIG treatments. HI injury caused an increase of C3b compared with sham-manipulated pups and IVIG treatment resulted in a highly significant suppression of $\mathrm{HI}$-induced increases in $\mathrm{C} 3 \mathrm{~b}$ levels, while vehicle had no effect on the $\mathrm{C} 3 \mathrm{~b}$ expression (Figure 4). The expression of $\mathrm{C} 3 \mathrm{~b}$ in injured vs. corresponding contralateral brain sites was evaluated by quantitative immunohistochemistry. The HI-induced increase in levels of C3b was significantly attenuated by IVIG but not vehicle treatment. The overexpression of $\mathrm{C} 3 \mathrm{~b}$ was confined to the site of injury, because the levels of $\mathrm{C} 3 \mathrm{~b}$ in samples from the corresponding contralateral side of the brain were 9-fold lower than in samples from the ipsilateral (infarcted) side and not significantly different from baseline values found in sham controls (Figure 5).

Table 1. Mortality rates in mice subjected to HI brain injury and different treatments

\begin{tabular}{lccc}
\hline Treatment & Died & Total \# & Percent \\
\hline Sham & 0 & 32 & 0.0 \\
HI alone & 9 & 42 & 21.2 \\
+Vehicle & 6 & 32 & 18.7 \\
+Pre-vehicle & 7 & 28 & 25.0 \\
+Pre-IVIG & 0 & 24 & 0.0 \\
\hline
\end{tabular}

An overall $\chi^{2}$ test value (for four degrees of freedom) for a 2-by-5 contingency table was $14.5, P<0.01$. Mortality rate in pre-IVIG group was significantly different from the pre-vehicle and vehicle control and not different from the sham group, as assessed by Fisher's exact test for pairwise comparisons.

$\mathrm{HI}$, hypoxic-ischemic; IVIG, intravenous immunoglobulin. 

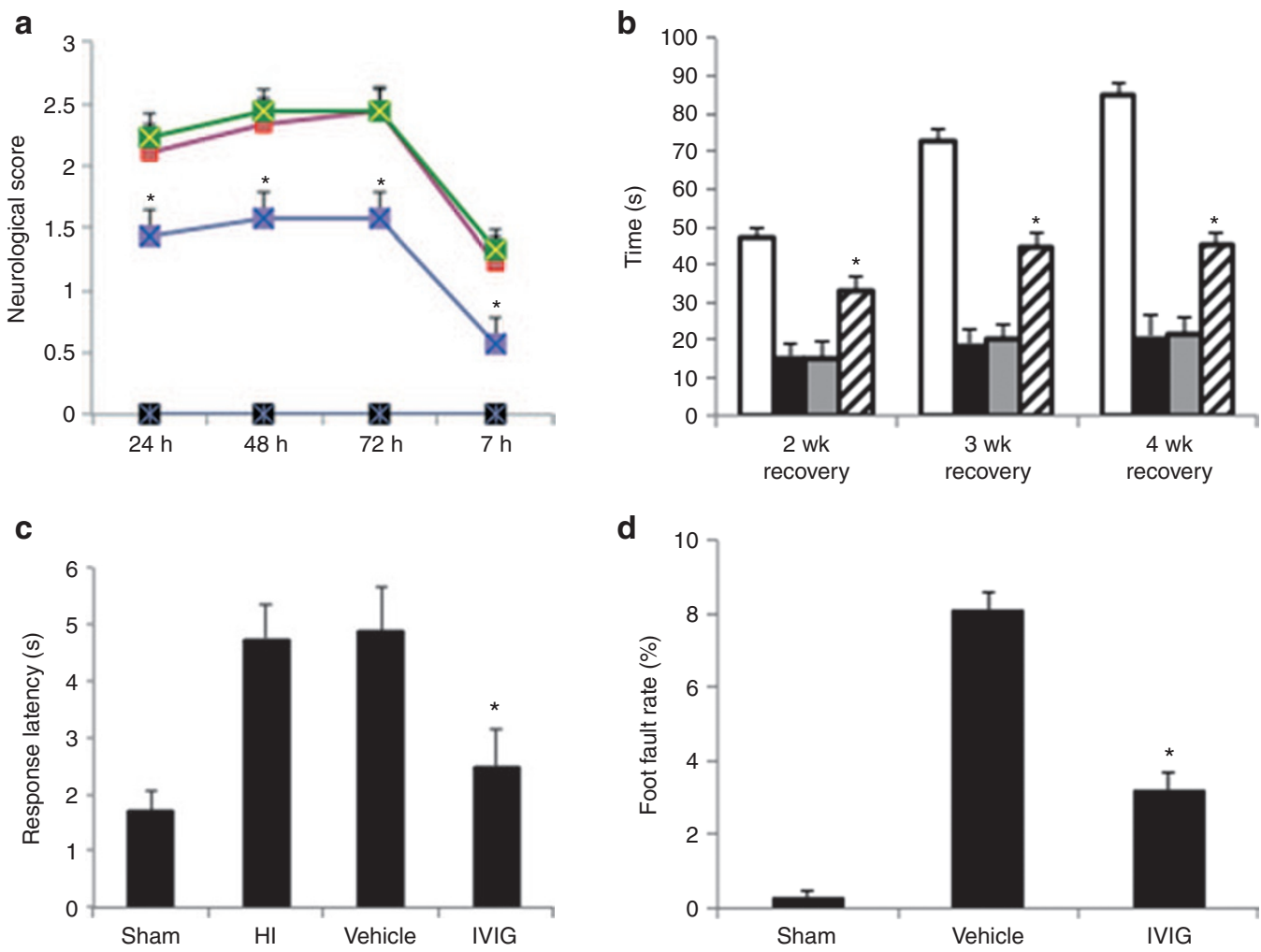

Figure 3. Attenuation of neurological deficits in intravenous immunoglobulin (IVIG)-treated pups subjected to hypoxic-ischemic (HI) injury. (a) Neurological deficit score in IVIG-treated pups (blue squares, $n=7$ ) at $24 \mathrm{~h}, 48 \mathrm{~h}, 72 \mathrm{~h}$, and $7 \mathrm{~d}$ after HI injury when compared with nontreated (red squares, $n=9$ ), vehicle-treated pups (green squares, $n=9$ ), and sham controls (black squares); ${ }^{*} P<0.05$ IVIG vs. vehicle at each time point. (b) Neurological deficit evaluated by wire hanging test in sham (no fill, $n=6$ ), HI-only (black columns, $n=9$ ), vehicle-treated (gray columns, $n=9$ ), and IVIG-treated (hatched columns, $n=7$ ) pups 2,3 , and 4 wk following $\mathrm{HI}$ injury; ${ }^{*} P<0.0001$ vs. vehicle. (c) The righting reflex latency response in pups treated with IVIG at $2 \mathrm{~g} /$ $\mathrm{kg}(\mathrm{IVIG}, n=8)$, nontreated pups (HI, $n=9$ ), and vehicle-treated animals (Vehicle, $n=9$ ); ${ }^{*} P<0.01$ vs. vehicle. (d) The foot fault rate in sham ( $\left.n=7\right)$, IVIGtreated $(n=9)$, and vehicle-treated $(n=8)$ pups; ${ }^{*} P<0.001$ vs. vehicle.

\section{DISCUSSION}

Our data provide, for the first time, preclinical evidence of neuroprotective effect of IVIG in the rat model of neonatal asphyxia or HIE. Both pre- and posttreatments with IVIG significantly reduced the size of brain infarction that was observed in nontreated animals subjected to HI injury. Furthermore, IVIG pre-treatment eliminated mortality when compared with vehicle-treated pups. Preservation of neurons had subsequent impact on functional outcome, which was markedly improved in IVIG-treated animals both early and several weeks after $\mathrm{HI}$ injury. In-situ complement activation triggered by $\mathrm{HI}$ and demonstrated by deposition of active complement fragments was attenuated by IVIG treatment.

Following initial resuscitation and stabilization, treatment of HIE is largely supportive (5). Intervention strategies aim to avoid any further brain injury in affected infants. Experimental data suggest that mild hypothermia $\left(3-4{ }^{\circ} \mathrm{C}\right.$ below baseline temperature) applied within a few hours (no later than $6 \mathrm{~h}$ ) of injury is neuroprotective. Induction of moderate hypothermia for $72 \mathrm{~h}$ in infants who had perinatal asphyxia resulted in improved neurologic outcomes in survivors (6). Recent metaanalysis data indicate that cooling reduces fatalities without an increase of disabilities in survivors and support institution of hypothermia in infants with moderate to severe HI encephalopathy before $6 \mathrm{~h}$ of age (7).
There were no attempts, in either experimental setting nor in human neonates, to attenuate harmful effect of active complement fragments in this condition. IVIG is known for its immunomodulatory and anti-inflammatory effects, but the mechanism has not been fully elucidated yet. Scavenging of harmful complement fragments appears to be one possible explanation. The theory of scavenging has been introduced based on data from in vitro $(8)$, in vivo $(9,10)$, and patient (11) studies, strongly suggesting that immunoglobulin molecules infused in supraphysiologic concentrations bind active complement fragments, thus preventing their interaction with specific receptors on target cells and subsequent immune damage. Immunoglobulin molecules engage different structural domains in the process of scavenging constant domain of the Fab region to neutralize $\mathrm{C} 3 \mathrm{a}$ and $\mathrm{C} 5 \mathrm{a}$ (12) and the Fc fragment to bind large activation products such as C3b and C4b (13). The proof of concept can be found in studies that employed IVIG treatment for different complement-mediated conditions. One of these was experimental stroke induced by transient occlusion of middle cerebral artery. IVIG, injected before or 1 and $3 \mathrm{~h}$ after brain ischemia, eliminated mortality and significantly reduced infarction size and the degree of neurological deficit in mice, even in relatively small doses $(0.5 \mathrm{~g} / \mathrm{kg})$. The effect appeared to be mediated by prevention of complement-mediated neuronal 
cell death (14). Recently, it was demonstrated that IVIG exerts its protective effect against ischemic brain injury by suppressing NLRP1 and NLRP3 inflammasome activity (15). It was also shown that IVIG might act directly on neurons to prevent damage in acute ischemic stroke by inhibiting cell death pathways and upregulating anti-apoptotic $\mathrm{Bcl} 2$ protein (16).

Considering significant anatomical, functional and survival rate improvements that were obtained by IVIG in rat pups subjected to $\mathrm{HI}$ injury, it would be justifiable to test the efficacy of IVIG in the treatment of human birth asphyxia as add-on to cooling. Such translation could be immediate, since IVIG is considered a safe therapeutic modality already in use for other indications and, as such, is readily available.

\section{METHODS}

\section{Animals}

Seven-day-old (P7) Wistar rat pups $(n=250)$ of both sexes, $15 \mathrm{~g}$ average body weight were used in this study. Experiments were performed in accordance to the US National Institutes of Health (Bethesda, MD) guidelines for humane treatment of animals and approved by the Institutional Animal Care and Use Committee of the University of Maryland School of Medicine.

\section{HI Brain Injury Model}

Animals were subjected to a modification of the rat model for HI that has been used extensively to identify mechanisms of brain injury resulting from perinatal $\mathrm{HI}$ and to test potential therapeutic interventions (17). In brief, animals were anesthetized by breathing halothane ( $4 \% \mathrm{v} / \mathrm{v}$ for induction and $1.5 \% \mathrm{v} / \mathrm{v}$ for maintenance) mixed with $37.5 \%$ nitrous oxide in oxygen. The left common carotid artery was
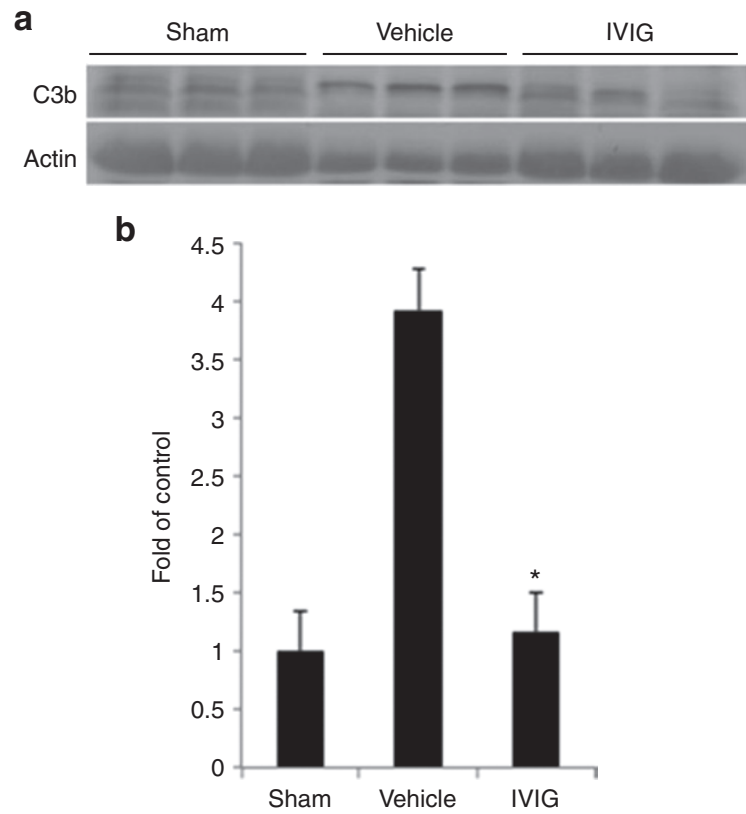

Figure 4. Intravenous immunoglobulin (IVIG) suppresses C3b at the site of brain infarction. (a) Proteins from ipsilateral cerebral cortex samples from mice subjected to sham surgery $(n=3)$, vehicle $(n=3)$, and IVIGtreatment at $2 \mathrm{~g} / \mathrm{kg}$ administered $1 \mathrm{~h}$ after hypoxic-ischemic $(\mathrm{HI})$ injury $(n=3)$ were immunoblotted by using anti-C3b and anti-actin antibodies. (b) C3b levels determined by densitometric analysis of immunoblots normalized to actin levels and expressed as a fold increase of the mean value for sham-treated mice in the infarcted brain region; ${ }^{*} P<0.01$ VIG vs. vehicle. Representative of four similar experiments.
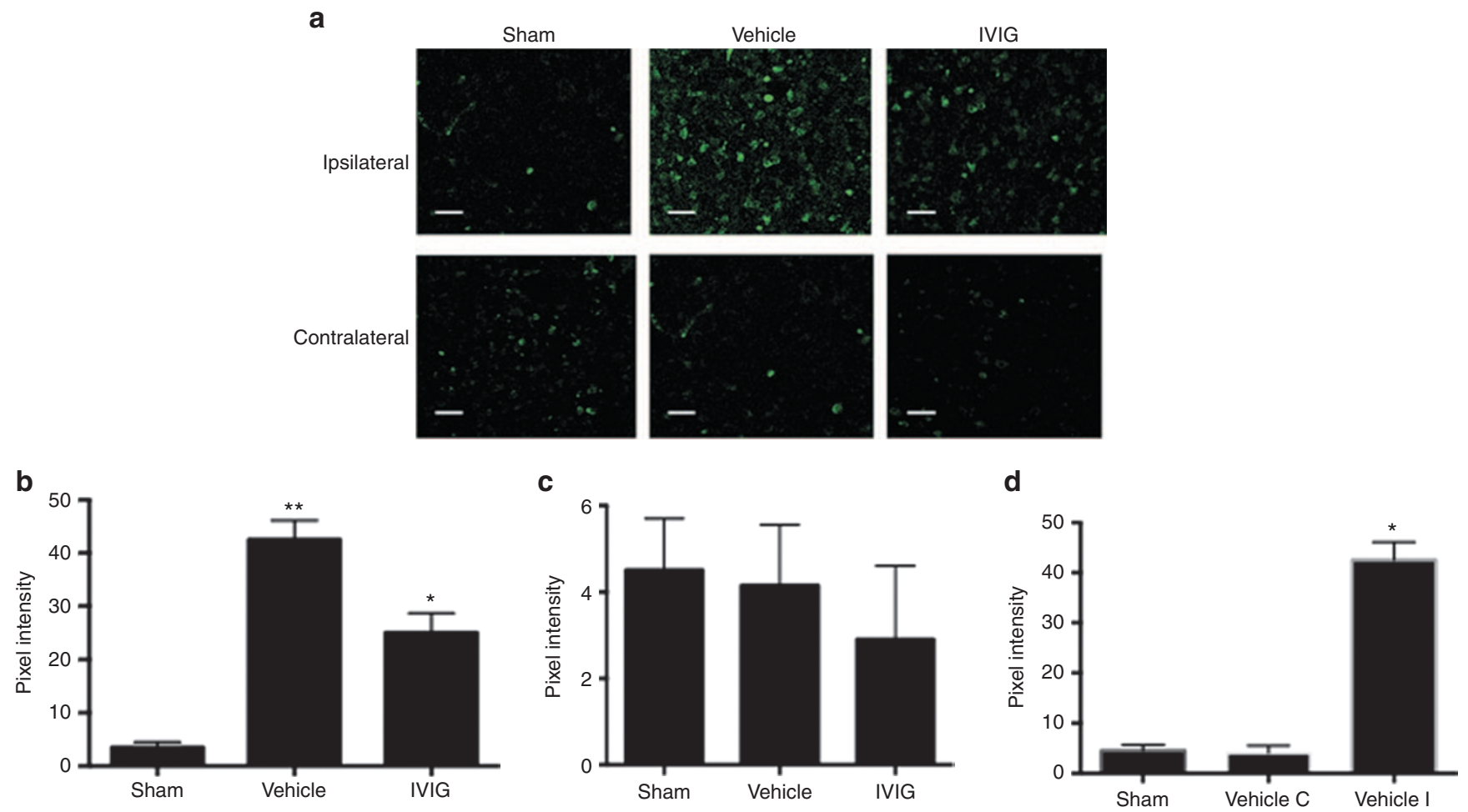

Figure 5. C3b expression in ipsilateral and contralateral brain sections. (a) Ipsilateral and contralateral brain sections from sham $(n=6)$, vehicle $(n=5)$, and intravenous immunoglobulin (IVIG)-treated $(n=5)$ pups were stained for C3b (green fluorescence). (b) C3b signal intensity comparison in the ipsilateral brain sections among sham $(n=4)$, vehicle $(n=4)$, and IVIG $(n=4)$ groups; ${ }^{* *} P<0.001$, vehicle vs. sham; ${ }^{*} P<0.01$ IVIG vs. vehicle. (c) Quantification of pixel intensity in brain samples from the contralateral hemisphere in sham $(n=4)$, vehicle $(n=4)$, and IVIG $(n=4)$, samples. (d) Comparison of C3b signals from ipsilateral (Vehicle I) and corresponding contralateral (Vehicle C) hemisphere; ${ }^{*} P<0.001$. Scale bar $=50 \mu \mathrm{m}$; magnification $40 \times$. Representative of three similar experiments. 
isolated, double-ligated with 7-0 silk sutures and cut between the ligatures. The pups were then placed in a chamber ventilated with warm humidified air $\left(35^{\circ} \mathrm{C}\right)$; after $30 \mathrm{~min}$ of breathing the air, hypoxia was introduced by switching to warm humidified $8 \%$ oxygen in nitrogen for $2 \mathrm{~h}$. In order to control for the variables such as anesthesia, surgical trauma and length of exposure to normal humidified air, pups were subjected to sham procedure that included anesthesia, dissection but not ligation of the left common carotid artery and ventilation with warmed humidified air for $2 \mathrm{~h}$.

\section{Treatment Regimen}

IVIG (Gammagard, $10 \%$ liquid) at $2 \mathrm{mg} / \mathrm{g}$ was infused to rat pups via catheterized common carotid artery either $30 \mathrm{~min}$ before induction of ischemia or $1 \mathrm{~h}$ following exposure to hypoxia. IVIG vehicle $(0.25 \mathrm{~mol} / \mathrm{l}$ glycine, $\mathrm{pH} 4.6-5.1)$ at the volume equivalent to that in which the intended dose of immunoglobulin molecules was delivered, was infused into control pups using the same intravascular route before and after HI injury. Operator inducing HI injury was blinded to the treatment arm.

\section{Brain Infarction Size Measurement}

The size of infarction was evaluated $2 \mathrm{~d}$ after HI injury, when animals were euthanized, their brains removed and cut into $2 \mathrm{~mm}$ coronal sections that were then stained for $20 \mathrm{~min}$ at $37^{\circ} \mathrm{C}$ with $2 \% 2,3,5$-triphenyltetrazolium chloride monohydrate which produces a dark red color upon binding to normal tissue; white areas represent infarctions or necrotic regions (Figure 1). The sections were scanned, and the infarction area in each section was calculated by subtracting the noninfarct area of the ipsilateral side from the area of the contralateral side (to correct for edema at the ipsilateral side) using NIH image analysis software (ImageJ, Bethesda, MD). The overall infarction size is shown as ratio (percent) of the ipsilateral hemispheric volume. All sections were coded to ensure blinded analysis.

\section{Neurological Deficit Evaluation}

Loss of neurological function was evaluated by a battery of tests, both early and up to 1 mo following HI brain injury. Neurological deficit score is based on a $0-4$ scale $((0$, no deficit; 1 , failure to extend right paw; 2 , circling to the right; 3 , falling to the right; 4 , unable to walk spontaneously) and was assessed at $24 \mathrm{~h}, 48 \mathrm{~h}, 72 \mathrm{~h}$ and $7 \mathrm{~d}$ after HI injury. For the righting reflex test (18), pups were placed on their backs, and the time required to turn over on all four feet and touch the platform (right themselves) was measured. The wire hang test (19) was used at 2, 3, and $4 \mathrm{wk}$ after HI injury. In this maneuver, pups were allowed to grasp by their four paws a $2-\mathrm{mm}$ diameter metal wire maintained horizontally $35 \mathrm{~cm}$ above a thick layer of soft bedding. The length of time until the mice fell from the wire was recorded. After each fall, the mice were allowed to recover for $1 \mathrm{~min}$. Each session consisted of three trials from which the scores were averaged. Foot fault or grid walk (20) is a test of motor coordination and was applied at $4 \mathrm{wk}$ after HI. Each animal was placed at one end of the grid suspended above a surface and monitored or videotaped from the side as they walk across the grid. The number of forelimb and hind limb placement errors as the animal traverses the grid is scored. An error is counted whenever a limb misses a bar and extends downward through the plane of the bars. Each functional test was performed in a blinded fashion.

\section{Gel Electrophoresis and Western Blotting}

Brain cortical samples were placed in lysis buffer containing 300 $\mathrm{mmol} / \mathrm{l} \mathrm{NaCl}, 1.5 \mathrm{mmol} / \mathrm{l} \mathrm{MgCl}, 25 \mathrm{mmol} / \mathrm{l} \mathrm{HEPES}, 20 \mathrm{mmol} / \mathrm{l}$ $\beta$-glycerophosphate, $0.2 \mathrm{mmol} / \mathrm{l}$ EDTA, $0.5 \mathrm{mmol} / 1$ dithiothreitol, and $0.1 \%$ Triton- $\mathrm{X}$ with protease inhibitors. Tissues were lysed by $30-\mathrm{s}$ sonication at $4{ }^{\circ} \mathrm{C}$ using an ultrasonic processor (VC $130 \mathrm{~PB}$, Sonic \& Materials, Newtown, CT) and the cellular extracts were centrifuged for $5 \mathrm{~min}$ at $18,000 \mathrm{~g}$. Protein content was then determined by a bicinchoninic acid assay kit from Bio-Rad (Hercules, CA). Supernatant samples $(50 \mu \mathrm{g}$ per lane) were separated on $10 \%$ sodium dodecyl sulfate gels and the resolved proteins electrophoretically transferred to a $0.45-\mu \mathrm{m}$ polyvinylidene fluoride membrane (Millipore, Bedford, $\mathrm{MA})$. The blots were incubated with a primary anti-C3b antibody goat polyclonal (Calbiochem, Billerica, CA) and anti- $\beta$ actin monoclonal antibody (Sigma Chemical, St Louis, MO) overnight at $4{ }^{\circ} \mathrm{C}$. Following incubation with horseradish peroxidase-conjugated secondary IgG for $1 \mathrm{~h}$, reaction products were visualized using the enhanced chemiluminescence assay (Amersham, Piscataway, NJ). Relative changes in protein expression were estimated from the mean pixel density of each protein band using the Scion Image program (Orem, UT).

\section{Immunohistochemical Evaluation}

Mice designated for immunohistochemical studies were deeply anaesthetized after $24 \mathrm{~h}$ of reperfusion, and transcardially perfused with phosphate-buffered saline until the outflow ran clear. Fixation was achieved with $4 \%$ paraformaldehyde in phosphate-buffered saline. Brains were removed and equilibrated in $30 \%$ sucrose in $0.1 \mathrm{~mol} / \mathrm{l}$ phosphate-buffered saline. Following blockade of nonspecific antibody binding, sections were incubated overnight with primary mouse IgM anti-rat C3b (Cedarlane, Burlington, NC) at $4{ }^{\circ} \mathrm{C}$. After being incubated with appropriate Alexa Fluor 488 (green fluorescence) conjugated secondary antibodies (Invitrogen, Carlsbad, CA) for $45 \mathrm{~min}$, images were acquired with an Olympus fluorescence microscope. Quantitation of pixel intensity was done using IP labs Software (BD biosciences, San Diego, CA) in a blinded fashion.

\section{Statistical Analysis}

Numerical values are expressed as mean \pm SEM. Statistical analysis of data related to brain infarction was performed using one-way analysis of variance followed by Newman-Keuls post hoc test for pairwise comparisons. Mortality rate comparisons were made by $\chi^{2}$ and Fisher's exact test. Neurological scores were analyzed by Kruskal-Wallis test and Dunn's Multiple Comparison Test. $P<0.05$ was considered to be significant. All statistical evaluation was performed using Prism 6.0 (GraphPad software, La Jolla, CA).

\section{STATEMENT OF FINANCIAL SUPPORT}

This study was supported by Investigator Initiated Study (IIS) Award from Baxter Healthcare (Westlake Village, CA).

Disclosure: B.C., B.H., and M.B. received monetary compensation from Baxter Healthcare proportionate to the percentage of time they invested into this study. The other author declared no conflict of interest.

\section{REFERENCES}

1. Bryce J, Boschi-Pinto C, Shibuya K, Black RE; WHO Child Health Epidemiology Reference Group. WHO estimates of the causes of death in children. Lancet 2005;365:1147-52.

2. Lawn J, Shibuya K, Stein C. No cry at birth: global estimates of intrapartum stillbirths and intrapartum-related neonatal deaths. Bull World Health Organ 2005;83:409-17.

3. Cowell RM, Plane JM, Silverstein FS. Complement activation contributes to hypoxic-ischemic brain injury in neonatal rats. J Neurosci 2003;23:945968.

4. Schultz SJ, Aly H, Hasanen BM, et al. Complement component 9 activation, consumption, and neuronal deposition in the post-hypoxic-ischemic central nervous system of human newborn infants. Neurosci Lett 2005;378:1-6.

5. Shankaran $S$. The postnatal management of the asphyxiated term infant. Clin Perinatol 2002;29:675-92.

6. Azzopardi DV, Strohm B, Edwards AD, et al.; TOBY Study Group. Moderate hypothermia to treat perinatal asphyxial encephalopathy. $\mathrm{N}$ Engl J Med 2009;361:1349-58.

7. Jacobs SE, Berg M, Hunt R, Tarnow-Mordi WO, Inder TE, Davis PG. Cooling for newborns with hypoxic ischaemic encephalopathy. Cochrane Database Syst Rev 2013;1:CD003311.

8. Basta M, Fries LF, Frank MM. High doses of intravenous Ig inhibit in vitro uptake of C4 fragments onto sensitized erythrocytes. Blood 1991;77:376-80.

9. Basta M, Langlois PF, Marques M, Frank MM, Fries LF. High-dose intravenous immunoglobulin modifies complement-mediated in vivo clearance. Blood 1989;74:326-33.

10. Basta M, Kirshbom P, Frank MM, Fries LF. Mechanism of therapeutic effect of high-dose intravenous immunoglobulin. Attenuation of acute, 
complement-dependent immune damage in a guinea pig model. J Clin Invest 1989;84:1974-81.

11. Basta M. Modulation of complement-mediated immune damage by intravenous immune globulin. Clin Exp Immunol 1996;104:Suppl 1:21-5.

12. Basta M, Van Goor F, Luccioli S, et al. F(ab)'2-mediated neutralization of C3a and C5a anaphylatoxins: a novel effector function of immunoglobulins. Nat Med 2003;9:431-8.

13. Basta M. Ambivalent effect of immunoglobulins on the complement system: activation versus inhibition. Mol Immunol 2008;45:4073-9.

14. Arumugam TV, Tang SC, Lathia JD, et al. Intravenous immunoglobulin (IVIG) protects the brain against experimental stroke by preventing complement-mediated neuronal cell death. Proc Natl Acad Sci USA 2007;104:14104-9.

15. Fann DY, Lee SY, Manzanero S, et al. Intravenous immunoglobulin suppresses NLRP1 and NLRP3 inflammasome-mediated neuronal death in ischemic stroke. Cell Death Dis 2013;4:e790.
16. Widiapradja A, Vegh V, Lok KZ, et al. Intravenous immunoglobulin protects neurons against amyloid beta-peptide toxicity and ischemic stroke by attenuating multiple cell death pathways. J Neurochem 2012;122: 321-32.

17. Vannucci RC, Connor JR, Mauger DT, et al. Rat model of perinatal hypoxic-ischemic brain damage. J Neurosci Res 1999;55: 158-63.

18. Fan LW, Lin S, Pang Y, et al. Hypoxia-ischemia induced neurological dysfunction and brain injury in the neonatal rat. Behav Brain Res 2005;165:8090.

19. Dorchies OM, Reutenauer-Patte J, Dahmane E, et al. The anticancer drug tamoxifen counteracts the pathology in a mouse model of duchenne muscular dystrophy. Am J Pathol 2013;182:485-504.

20. Silvestrin RB, de Oliveira LF, Batassini C, Oliveira A, e Souza TM. The footfault test as a screening tool in the 6-hydroxydopamine rat model of Parkinson's disease. J Neurosci Methods 2009;177:317-21. 